Ecology, 97(3), 2016, pp. 796-798

(C) 2016 by the Ecological Society of America

\section{A simple Bayesian method of inferring extinction: comment}

Andrew R. Solow ${ }^{1}$

Understanding the timing of extinction events is of interest to both paleobiologists concerned with macroevolutionary processes and patterns and ecologists concerned with the dynamics and conservation of threatened populations. In both cases, it is common that the only information available for inference about the extinction time of a species is a record of its sightings. For ancient species, these sightings typically consist of fossil finds, while for modern species they may consist of live or recently live specimens, other kinds of physical evidence, or visual sightings.

Numerous methods have been proposed for inference about extinction time based on a sighting record in both the paleobiological literature (e.g., Strauss and Sadler 1989, Solow 1996) and the ecological literature (e.g., Solow 1993, Rivadeneira et al. 2009). These methods differ in the underlying statistical model of the sighting record and also in whether a Bayesian or non-Bayesian approach is taken. The purpose of this note is to comment on a Bayesian method recently proposed by Alroy (2014). In developing this method, Alroy eschewed the usual approach of laying out a statistical model and applying Bayesian tools to it in favor of less formal exposition. This is not by itself a problem, but it can leave a reader (like me) somewhat confused. Here, I will return to the usual approach to clarify what Alroy's method is aimed at, to raise some technical issues with its implementation, and to provide a standard Bayesian approach to the same problem that avoids these issues.

The situation considered by Alroy is as follows. A time interval (or stratigraphic section) is divided into equal subintervals $1,2, \ldots$. The unknown extinction time $\tau_{E}$ is defined as the earliest subinterval following extinction. Let $\tau_{j}$ be the event that $\tau_{E}=j$ with prior probability $\pi_{j}$, and let $T_{j}$ be the event that $\tau_{E} \leq j$. Each subinterval is observed to contain either no sighting or at least one sighting. I will refer to the latter subintervals as occupied. The probability that

Manuscript received 22 February 2015; revised 16 June 2015; accepted 29 June 2015. Corresponding Editor: P. de Valpine.

${ }^{1}$ Woods Hole Oceanographic Institution, Woods Hole, Massachusetts, 02543, USA. E-mail: asolow@whoi.edu subinterval $j$ is occupied is $p$ if $j<\tau_{E}$ and 0 if $j \geq \tau_{E}$. Let $m$ be the most recent occupied subinterval. Finally, let $s_{j}$ be the partial sighting record through subinterval $j$.

Alroy was interested in finding the posterior probability of $\tau_{m+j}$ (or $T_{m+j}$ ) given $s_{m+j}$. To lay out a standard Bayesian approach, I will begin by focusing in some detail on the case $j=1$. By Bayes' Theorem

$$
\operatorname{pr}\left(\tau_{m+1} \mid s_{m+1}\right)=\frac{\operatorname{pr}\left(s_{m+1} \mid \tau_{m+1}\right) \pi_{m+1}}{\operatorname{pr}\left(s_{m+1}\right)} .
$$

The partial sighting record $s_{m+1}$ is the augmentation of $s_{m}$ by the event $u_{m+1}$ that subinterval $m+1$ is unoccupied, so that

$$
\operatorname{pr}\left(s_{m+1} \mid \tau_{m+1}\right)=\operatorname{pr}\left(s_{m} \mid \tau_{m+1}\right) \operatorname{pr}\left(u_{m+1} \mid \tau_{m+1}\right) .
$$

Also:

$$
\begin{aligned}
\operatorname{pr}\left(s_{m+1}\right) & =\sum_{k=1}^{\infty} \operatorname{pr}\left(s_{m+1} \mid \tau_{k}\right) \pi_{k} \\
& =\sum_{k=m+1}^{\infty} \operatorname{pr}\left(s_{m+1} \mid \tau_{k}\right) \pi_{k} \\
& =\sum_{k=m+1}^{\infty} \operatorname{pr}\left(s_{m} \mid \tau_{k}\right) \operatorname{pr}\left(u_{m+1} \mid \tau_{k}\right) \pi_{k} .
\end{aligned}
$$

Noting that $\operatorname{pr}\left(s_{m} \mid \tau_{k}\right)=\operatorname{pr}\left(s_{m} \mid \tau_{m+1}\right)$ for all $k \geq m+1$ and that $\operatorname{pr}\left(u_{m+1} \mid \tau_{k}\right)=1$ if $k=m+1$ and $1-p$ if $k>m+1$, it follows that

$$
\operatorname{pr}\left(\tau_{m+1} \mid s_{m+1}\right)=\frac{\pi_{m+1}}{\pi_{m+1}+(1-p) \sum_{k=2}^{\infty} \pi_{m+k}}
$$

Retaining my notation $p$ for the pre-extinction persubinterval sighting probability, Alroy (2014 p. 587) gave the result

$$
\varepsilon_{1}=\frac{E}{E+(1-p)(1-E)}
$$

with $\varepsilon_{1}$ and $E$ described as the posterior and prior probability of $\tau_{m+1}$. This agrees with Eq. 4 if $E$ is the conditional probability $\operatorname{pr}\left(\tau_{m+1} \mid \tau_{E}>m\right)$. Note that, because it involves $m$, Alroy's prior probability $E$ is posterior to the sighting record.

By the same argument leading to Eq. 4, it can be shown that

$$
\begin{aligned}
& \operatorname{pr}\left(T_{m+j} \mid s_{m+j}\right)= \\
& \frac{\sum_{k=1}^{j}(1-p)^{k-1} \pi_{m+k}}{\sum_{k=1}^{j}(1-p)^{k-1} \pi_{m+k}+(1-p)^{j} \sum_{k=j+1}^{\infty} \pi_{m+k}} .
\end{aligned}
$$

With the above interpretation of his notation, this expression produces the same result as the iterative 
calculation in Alroy (2014: 587). The posterior probability in Eq. 6 is sequential in the sense that it is conditional only on the observations through subinterval $m+j$. In a retrospective setting involving inference about $\tau_{E}$ based on the entire sighting record, the probability of interest is

$$
\begin{aligned}
& \operatorname{pr}\left(T_{m+j} \mid s_{m+T}\right)= \\
& \frac{\sum_{k=1}^{j}(1-p)^{k-1} \pi_{m+k}}{\sum_{k=1}^{T}(1-p)^{k-1} \pi_{m+k}+(1-p)^{T} \sum_{k=T+1}^{\infty} \pi_{m+k}}
\end{aligned}
$$

where $m+j$ is the last subinterval before the present. The posterior probabilities in Eqs 6 and 7 only coincide when $j=T$. This reflects the fact that the entire sighting record provides information about extinction at or before $m+j$ beyond that in the partial sighting record up to $m+j$.

As a simple example of the difference between sequential and retrospective results, the years in which sightings of the Dodo (Raphus cucullatus) were made on an island off Mauritius are 1598, 1601, 1602, 1607, 1611, 1628, 1631, 1638, and 1662 (Roberts and Solow 2003) and suppose that interest centers on the posterior probability that extinction occurred no later than 1672 (i.e., $10 \mathrm{yr}$ after the last sighting). Take 1598 as the beginning of the observation period and omit the initial sighting from the record. The corresponding values of $m$ and $T$ are 64 and 353, respectively, and the event of interest is $T_{m+10}$. Take the values of $p$ to be 0.11 and $\pi_{j}=\frac{1}{m+T}=0.0024, j=1,2, \ldots, m+T$. From Eq. 6, the sequential posterior probability $\operatorname{pr}\left(T_{m+10} \mid S_{m+10}\right)$ is 0.055 . From Eq. 7, the retrospective posterior probability $\operatorname{pr}(T-$ $\left.{ }_{m+10} \mid S_{m+T}\right)$ is 0.688 . The former is what a person in 1672 would have concluded about the extinction of the Dodo, while the latter is what a person today would conclude about the extinction of the Dodo by 1672 . I will return to this example below using a more appropriate prior distribution for extinction time.

This discussion is intended as a clarification of what Alroy did and not a criticism of the sequential approach. There certainly are situations in which the sequential approach is called for; for example, in monitoring a conservation or pest eradication program. However, in cases (such as the Dodo) where the entire sighting record is available, a good reason must be had not to use it.

I will now turn to Alroy's implementation of the sequential approach. The discussion applies equally to the retrospective approach. These calculations require the specification of the pre-extinction sighting probability $p$. Alroy (2014: 286) proposed estimating $p$ by the proportion of subintervals between the earliest and latest occupied subintervals that are occupied, and then treating the estimate as correct. For the Dodo sighting record, this estimate is $\frac{7}{63}=0.11$. This approach fails to account for uncertainty in $p$. As a practical matter, ignoring this uncertainty will not be a problem when it is small, but this is not always the case. In inference about extinction, $p$ is a so-called nuisance parameter. A standard approach, common to both Bayesian and non-Bayesian statistics, is to eliminate $p$ by conditioning on the number $n$ of occupied subintervals in the sighting record, this number being a sufficient statistic for $p$ (e.g., Reid 1995). Conditional on $n$, the likelihood is given by

$$
\operatorname{pr}\left(s_{m+j} \mid n, \tau_{m+j}\right)=\left(\begin{array}{c}
m+j-1 \\
n
\end{array}\right)^{-1} .
$$

The combinatorial term in Eq. 8 is the number of ways that $n$ of $m+j-1$ pre-extinction subintervals can be occupied, these ways being equally likely. It follows that the posterior sequential extinction probability conditional on $n$ is

$$
\begin{gathered}
\operatorname{pr}\left(T_{m+j} \mid n, s_{m+j}\right)= \\
\frac{\sum_{k=1}^{j}\left(\begin{array}{c}
m+k-1 \\
n
\end{array}\right)^{-1} \pi_{m+k}}{\sum_{k=1}^{j}\left(\begin{array}{c}
m+k-1 \\
n
\end{array}\right)^{-1} \pi_{m+k}+\left(\begin{array}{c}
m+j \\
n
\end{array}\right)^{-1} \sum_{k=j+1}^{\infty} \pi_{m+k}}
\end{gathered}
$$

In the example involving the Dodo, the use of Eq. 9 instead of Eq. 6 increases the posterior probability only slightly to 0.057 .

A more interesting issue concerns the prior distribution of extinction time. As noted, Alroy worked with the posterior distribution of $\tau_{E}$ given that $\tau_{E}>m$. He took this distribution to be exponential and chose the parameter to ensure that $\operatorname{pr}\left(\tau_{E}<m+r \mid \tau_{E}>m\right)=0.5$, where $r$ is the observed range (Alroy 2014: 586) or an estimate of the true range (Alroy 2014: 587) or twice an estimate of the true range (Alroy 2014: 588) of the species. Alroy's approach here is unusual in the sense that it does not separate the prior specification from the data. More importantly, although it appears to be connected to the notion of a non-informative prior, he gave no clear justification for this choice of the exponential parameter.

The standard Bayesian approach in this situation is to adopt a prior distribution for extinction time conditional on the value of a parameter and then to integrate this conditional prior distribution over a prior distribution for the parameter. This is the essence of the so-called hierarchical Bayes approach. As time or stratigraphic position is treated here as discrete, it is natural to take the conditional prior distribution of $\tau_{j}$ to be geometric so that

$$
\operatorname{pr}\left(\tau_{j} \mid q\right)=q(1-q)^{j-1}
$$

where $q$ is the per-subinterval extinction probability. In the absence of prior information, it is also natural to adopt a uniform distribution for $q$ with probability density function: 


$$
f(q)=10<q<1 .
$$

The sense in which this is non-informative is clear: a priori, all possible values of $q$ are equally likely. It follows that

$$
\pi_{j}=\int_{0}^{1} \operatorname{pr}\left(\tau_{j} \mid q\right) f(q) d q=1 /(j(j+1))
$$

which is a special case of the Yule-Simon distribution (Simon 1955). Other choices of $f(q)$ or, more generally, of specifying $\pi_{j}$ are also possible.

Returning to the example of the Dodo, in this case, $r=64$, and Alroy's method takes $\pi_{m+j}=0.011 \exp (-0.011(m+j))$ : or, by virtue of the memoryless property of the exponential distribution, $\operatorname{pr}\left(\tau_{m+j} \mid \tau_{E}<m\right)=0.011 \exp (-0.011 j)$. The corresponding posterior probability $\operatorname{pr}\left(T_{10} \mid n, s_{m+10}\right)$ given by Eq. 9 is 0.19 . If instead, the standard Bayesian approach is used, $\pi_{m+j}=1 /((m+j)(m+j+1))$ and $\operatorname{pr}\left(T_{10} \mid n, s_{m+10}\right)$ is 0.28 . It cannot be said that one of these is right and the other wrong. The point is that the choice of prior distribution matters and the one in Eq. 12 has a clear justification.

In summary, to a reader lacking Alroy's intuition, the method he proposed is something of a black box. The purpose of this comment has been to open this box. Doing so has clarified (a) that this method produces sequential extinction probabilities; (b) that it treats an estimate of the sighting probability as correct; (c) that the prior distribution of extinction time on which it is based is actually posterior to the sighting record, and (d) that the specification of this distribution is without a clear justification. In contrast, the standard Bayesian approach (the results of which are summarized in Eqs 9 and 12) addresses the same problem in a straightforward and transparent way, and there is something to be said for sticking with it unless it can be shown to be inferior.

\section{Literature Cited}

Alroy, J. 2014. A simple Bayesian method of inferring extinction. Paleobiology 40:584-607.

Reid, N. 1995. The roles of conditioning in inference. Statistical Science 10:138-157.

Rivadeneira, M. M., G. Hunt, and K. Roy. 2009. The use of sighting records to infer extinction. Ecology 90:1291-1300.

Roberts, D. L., and A. R. Solow. 2003. Flightless birds: when did the dodo go extinct? Nature 426:245.

Simon, H. A. 1955. On a class of skew distribution functions. Biometrika 42:425-440.

Solow, A. R. 1993. Inferring extinction from sighting data. Ecology 74:962-964.

Solow, A. R. 1996. Tests and confidence intervals for a common upper endpoint $\mathrm{n}$ fossil taxa. Paleobiology 22:406-410.

Strauss, D., and P. M. Sadler. 1989. Classical confidence intervals and Bayesian probability estimates for ends of local taxon ranges. Mathematical Geology 21:411-427.
Ecology, 97(3), 2016, pp. 798-800

(C) 2016 by the Ecological Society of America

\section{A simple Bayesian method of inferring extinction: reply}

\section{JOHN Alroy ${ }^{1}$}

Corresponding Editor: P. de ValpineThe purpose of Solow (2016) is to comment on a recently published method of mine (Alroy 2014). It also presents two new Bayesian methods of inferring extinction. These methods, like several others, are based on ecological or paleontological observations such as the monk seal sightings reported by Solow (1993a) or the dodo sightings listed by Roberts and Solow (2003).

Solow first proposes a retrospective equation (number 7) that uses fixed per-interval sampling probabilities derived from the observed data. Solow goes on to develop a second, sequential equation (number 9) that is instead based on combinatorial computations. He notes that in his view a "good reason" must be given to use sequential inference - i.e., inference based only on the data observed up to a time interval in the past that is of interest - when an entire sighting record could be used instead.

Neither of the new equations produce superior results.

This fact can be shown by applying Solow's equations to two Monte Carlo simulation data sets reported by Alroy (2014), each of which summarized histories of 1,000 species observed across 50 time intervals. Extinction was assumed to be a geometric process. In one trial of interest (Alroy 2014, Fig. 3A), the per-interval sighting rate was $20 \%$ and the per-interval extinction rate was $5 \%$. The latter rate was selected in order to avoid having all species go extinct during the trial and to create enough extinctions for the process to be visible. In another trial (Alroy 2014, Fig. 3C), the sighting rate was instead 50\% but there was no extinction (making it possible to test for false positives).

Solow's two equations perform very differently (Fig. 1). When cumulative counts are employed, equation 7 appears to be generally more accurate than the others given ongoing extinction (Fig. 1A). It is shown to be extremely aggressive at the end of the time series, however, even when there is no extinction (compare Fig. 1A,B). The reason that equation 7 appears to work well here is that it nearly amounts to declaring a species extinct as soon as it is last

Manuscript received 21 February 2015; revised 19 November 2015; accepted 29 December 2015; final version received 20 January 2016 Corresponding Editor: P. de Valpine.

${ }^{1}$ Department of Biological Sciences, Macquarie University, Sydney, NSW 2109, Australia. . E-mail: john.alroy@mq.edu.au 